\title{
A NOTE ON SOLVABILITY OF THREE-POINT BOUNDARY VALUE PROBLEMS FOR THIRD-ORDER DIFFERENTIAL EQUATIONS WITH $p$-LAPLACIAN
}

\author{
XINGYUAN LIU AND YUJI LIU
}

Abstract. Third-point boundary value problems for third-order differential equation

$$
\left\{\begin{array}{l}
{\left[q(t) \phi\left(x^{\prime \prime}(t)\right)\right]^{\prime}+k x^{\prime}(t)+g\left(t, x(t), x^{\prime}(t)\right)=p(t), t \in(0,1),} \\
x^{\prime}(0)=x^{\prime}(1)=x(\eta)=0
\end{array}\right.
$$

is considered. Sufficient conditions for the existence of at least one solution of above problem are established. Some known results are improved.

\section{Introduction}

In [1], the solvability of problem

$$
\left\{\begin{array}{l}
x^{\prime \prime \prime}(t)+k^{2} x^{\prime}(t)+g\left(x, x^{\prime}\right)=p(t), t \in(0, \pi), \\
x^{\prime}(0)=x^{\prime}(\pi)=x(\eta)=0
\end{array}\right.
$$

was studied, where $0<\eta<\pi$ and $R^{2}$;

$\left(B_{1}\right) . g(u, v)$ is continuous in $R^{2}$, has continuous partial derivatives $g_{u}$ and $g_{v}$, bounded in

$\left(B_{2}\right) .\left|1-g_{\nu}\right|+\sqrt{\pi\left|g_{u}\right|} \leq 2 k$ for $k \in N \backslash\{1\},\left|1-g_{\nu}\right|+\sqrt{\pi}\left|g_{u}\right| \leq 2$ for $k=1$, and $\lim _{|v| \rightarrow \infty} v g(u, v)=$ $\mu \neq 0$ uniformly for $u \in R$.

Problem (1) when $k=1$ was also studied by Nagle and Pothoven [2] and by Rovderova [4] under the condition

$\left(B_{3}\right) . g$ is bounded on one side and continuous.

In [3], Gupta studied the existence of solutions to boundary value problems similar to (1) of the type

$$
\left\{\begin{array}{l}
x^{\prime \prime \prime}(t)+\pi^{2} x^{\prime}(t)+g\left(t, x, x^{\prime}, x^{\prime \prime}\right)=p(t), t \in(0,1), \\
x^{\prime}(0)=x^{\prime}(1)=x(\eta)=0,
\end{array}\right.
$$

Received June 22, 2006.

2000 Mathematics Subject Classification. 34B15.

Key words and phrases. Solution, Three-point boundary value problem, Third order differential equation with $p$-Laplacian.

The author is supported by the Science Foundation of Educational Committee of Hunan Province and the National Natural Science Foundation of P.R.China. 
where $g$ is a Caratheodory function and $p \in L^{1}[0,1]$, under the conditions

(C). $\int_{0}^{1} p(t) \sin \pi t d t=0$, and $g$ satisfies

$$
g(t, u, v, w) v \geq 0, \text { for } t \in[0,1], u, v, w \in R
$$

and

$$
\lim _{|\nu| \rightarrow \infty} \frac{g(t, u, v, w)}{v}<3 \pi^{2} \text { uniformly for }(t, u, w) \in[0,1] \times R^{2} .
$$

The purpose of this paper is to establish new sufficient conditions for the existence of at least one solution of problem

$$
\left\{\begin{array}{l}
{\left[q(t) \phi\left(x^{\prime \prime}(t)\right)\right]^{\prime}+k x^{\prime}(t)+g\left(t, x(t), x^{\prime}(t)\right)=p(t), \quad t \in(0,1),} \\
x^{\prime}(0)=x^{\prime}(1)=x(\eta)=0,
\end{array}\right.
$$

where $k \in R, q, p \in L^{1}[0,1]$ and $g$ is a Caratheodory function, $\phi(x)=|x|^{p-2} x$ for $x \neq 0$ and $\phi(0)=0$, which is called $p$-Laplacian.

In Section 2, we establish existence results for problem (3). The examples, which can not be solved by result in [1-3], to illustrate the main theorems will be given in Section 4 .

\section{Main Result}

Let $X=C^{1}[0,1] \times C^{0}[0,1], Y=L^{1}[0,1] \times L^{1}[0,1]$, their norms are defined by $\|(x, y)\|=$ $\max \left\{\|x\|_{\infty},\left\|x^{\prime}\right\|_{\infty},\|y\|_{\infty}\right\}$ for $(x, y) \in X$ and $\|(u, v)\|=\max \left\{\int_{0}^{1}|u(s)| d s, \int_{0}^{1}|v(s)| d s\right\}$ for $(u, v) \in$ $Y$, respectively. Then $X$ and $Y$ are Banach spaces.

Let $D(L)=\left\{(x, y) \in X: x^{\prime \prime} \in L^{1}(0,1),(q y)^{\prime} \in L^{1}(0,1)\right.$ with $\left.x^{\prime}(0)=x^{\prime}(1)=x(\eta)=0\right\}$. Define the linear operator $L: D(L) \cap X \rightarrow Y$ by

$$
L\left(\begin{array}{l}
x(t) \\
y(t)
\end{array}\right)=\left(\begin{array}{l}
x^{\prime \prime}(t) \\
(q(t) y(t))^{\prime}
\end{array}\right) \text { for all }(x, y) \in D(L) \cap X
$$

Define the nonlinear operator $N: X \rightarrow Y$ by

$$
N\left(\begin{array}{l}
x(t) \\
y(t)
\end{array}\right)=\left(\begin{array}{l}
\phi^{-1}(y(t)) \\
-k x^{\prime}(t)-g\left(t, x(t), x^{\prime}(t)\right)+p(t)
\end{array}\right) \text { for all }(x, y) \in X .
$$

It is easy to show that $\operatorname{Ker} L=\{(0, c / q(t)): c \in R\} ; \operatorname{Im} L=\left\{(u, v) \in Y: \int_{0}^{1} u(s) d s=0\right\} ; L$ is a Fredholm operator of index zero; There are projectors $P: X \rightarrow X$ and $Q: Y \rightarrow Y$ such that $\operatorname{Ker} L=\operatorname{Im} P$ and $\operatorname{Ker} Q=\operatorname{Im} L$. In fact, we have

$$
\begin{aligned}
P\left(\begin{array}{l}
x(t) \\
y(t)
\end{array}\right) & =\left(\begin{array}{l}
0 \\
y(0) / q(t)
\end{array}\right) \text { for }(x, y) \in X \\
Q\left(\begin{array}{l}
u(t) \\
v(t)
\end{array}\right) & =\left(\begin{array}{l}
\int_{0}^{1} u(t) d t \\
0
\end{array}\right) \text { for }(u, v) \in Y
\end{aligned}
$$


Furthermore, let $\Omega \subset X$ be an open bounded subset with $\bar{\Omega} \cap D(L) \neq \varnothing$, then $N$ is $L$-compact on $\bar{\Omega} ;(x, y) \in D(L)$ is a solution of the operator equation $L(x, y)=N(x, y)$ implies that $x$ is a solution of problem (3).

Suppose

$\left(A_{1}\right)$. There are bounded functions $a \in L^{1}(0,1)$, bounded function $b \in L^{1}(0,1)$ and function $r \in L^{1}(0,1)$ such that $g(t, x, y)=h(t, x, y)+f(t, x, y)$, and there are positive constants $\beta, \theta$ such that $h(t, x, y) y \leq-\beta|y|^{\theta+1}$, and $|f(t, x, y)| \leq a(t)|x|^{\theta}+b(t)|y|^{\theta}+r(t)$, for all $(x, y) \in R^{2}$ and a.e. $t \in[0,1]$.

$\left(A_{2}\right)$. If $\theta>1$, then

$$
\|b\|_{\infty}+\left(\int_{0}^{1}|a(t)|^{\frac{\theta+1}{\theta}} d t\right)^{\frac{\theta}{\theta+1}}<\beta
$$

if $\theta=1$, then

$$
k+\|b\|_{\infty}+\left(\int_{0}^{1}|a(t)|^{2} d t\right)^{\frac{1}{2}}<\beta .
$$

$\left(A_{3}\right)$. There is a constant $\delta>0$ such that $q(t) \geq \delta$ for all $t \in[0,1]$.

Now, consider $L(x, y)=\lambda N(x, y)$ with $\lambda \in(0,1)$. We have

$$
\left\{\begin{array}{l}
x^{\prime \prime}(t)=\lambda \phi^{-1}(y(t)) \\
(q(t) y(t))^{\prime}=\lambda\left(-k x^{\prime}(t)-g\left(t, x, x^{\prime}\right)+p(t)\right) .
\end{array}\right.
$$

It follows that

$$
\left[q(t) \phi\left(x^{\prime \prime}(t)\right)\right]^{\prime}=\phi(\lambda) \lambda\left(-k x^{\prime}(t)-g\left(t, x, x^{\prime}\right)+p(t)\right) .
$$

Thus

$$
\left[q(t) \phi\left(x^{\prime \prime}(t)\right)\right] x^{\prime}(t)=\phi(\lambda) \lambda\left(-k x^{\prime}(t)-g\left(t, x, x^{\prime}\right)+p(t)\right) x^{\prime}(t) .
$$

Since $x^{\prime}(0)=x^{\prime}(1)=0$ implies that

$$
\int_{0}^{1}\left[q(t) \phi\left(x^{\prime \prime}(t)\right)\right]^{\prime} x^{\prime}(t) d t=-\int_{0}^{1} q(t) \phi\left(x^{\prime \prime}(t)\right) x^{\prime \prime}(t) d t \leq 0,
$$

we get

$$
-k \int_{0}^{1}\left[x^{\prime}(t)\right]^{2} d t+\int_{0}^{1} p(t) x^{\prime}(t) d t \leq \int_{0}^{1} g\left(t, x(t), x^{\prime}(t)\right) x^{\prime}(t) d t .
$$

$\left(A_{1}\right)$ implies that

$$
\begin{aligned}
\beta \int_{0}^{1}\left|x^{\prime}(t)\right|^{\theta+1} d t \leq & \left.k \int_{0}^{1}\left[x^{\prime}(t)\right]^{2} d t-\int_{0}^{1} p(t)\right) x^{\prime}(t) d t+\int_{0}^{1} f\left(t, x(t), x^{\prime}(t)\right) x^{\prime}(t) d t \\
\leq & k \int_{0}^{1}\left[x^{\prime}(t)\right]^{2} d t+\int_{0}^{1}|p(t)|\left|x^{\prime}(t)\right| d t+\int_{0}^{1}|a(t)||x(t)|^{\theta}\left|x^{\prime}(t)\right| d t \\
& +\int_{0}^{1}\left|b ( t ) \left\|\left.x^{\prime}(t)\right|^{\theta+1} d t+\int_{0}^{1}\left|r(t) \| x^{\prime}(t)\right| d t\right.\right. \\
\leq & k \int_{0}^{1}\left[x^{\prime}(t)\right]^{2} d t
\end{aligned}
$$


98

XINGYUAN LII AND YUJI LIU

$$
\begin{aligned}
& +\left(\int_{0}^{1}(|p(t)|+|r(t)|)^{\frac{\theta+1}{\theta}} d t\right)^{\frac{\theta}{\theta+1}}\left(\int_{0}^{1}\left|x^{\prime}(t)\right|^{\theta+1} d t\right)^{\frac{1}{\theta+1}} \\
& +\|b\|_{\infty} \int_{0}^{1}\left|x^{\prime}(t)\right|^{\theta+1} d t+\int_{0}^{1}\left|a(t) \| x^{\prime}(t)\right| d t\left(\int_{0}^{1}\left|x^{\prime}(t)\right| d t\right)^{\theta} \\
\leq & k \int_{0}^{1}\left[x^{\prime}(t)\right]^{2} d t \\
& +\left(\int_{0}^{1}(|p(t)|+|r(t)|)^{\frac{\theta+1}{\theta}} d t\right)^{\frac{\theta}{\theta+1}}\left(\int_{0}^{1}\left|x^{\prime}(t)\right|^{\theta+1} d t\right)^{\frac{1}{\theta+1}} \\
& +\|b\|_{\infty} \int_{0}^{1}\left|x^{\prime}(t)\right|^{\theta+1} d t \\
& +\left(\int_{0}^{1}|a(t)|^{\frac{\theta+1}{\theta}} d t\right)^{\frac{\theta}{\theta+1}}\left(\int_{0}^{1}\left|x^{\prime}(t)\right|^{\theta+1} d t\right)^{\frac{1}{\theta+1}}\left(\int_{0}^{1}\left|x^{\prime}(t)\right|^{\theta+1} d t\right)^{\frac{\theta}{\theta+1}} \\
\leq & k \int_{0}^{1}\left[x^{\prime}(t)\right]^{2} d t \\
& +\left(\int_{0}^{1}(|p(t)|+|r(t)|)^{\frac{\theta+1}{\theta}} d t\right)^{\frac{\theta}{\theta+1}}\left(\int_{0}^{1}\left|x^{\prime}(t)\right|^{\theta+1} d t\right)^{\frac{1}{\theta+1}} \\
& +\|b\|_{\infty} \int_{0}^{1}\left|x^{\prime}(t)\right|^{\theta+1} d t \\
& +\left(\int_{0}^{1}|a(t)|^{\frac{\theta+1}{\theta}} d t\right)^{\frac{\theta}{\theta+1}} \int_{0}^{1}\left|x^{\prime}(t)\right|^{\theta+1} d t .
\end{aligned}
$$

If $\theta=1$, then

$$
\begin{gathered}
\left(\beta-k-\|b\|_{\infty}-\left(\int_{0}^{1}|a(t)|^{2} d t\right)^{\frac{1}{2}}\right) \int_{0}^{1}\left|x^{\prime}(t)\right|^{2} d t \\
\leq\left(\int_{0}^{1}(|p(t)|+|r(t)|)^{2} d t\right)^{\frac{1}{2}}\left(\int_{0}^{1}\left|x^{\prime}(t)\right|^{2} d t\right)^{\frac{1}{2}} .
\end{gathered}
$$

If $\theta>1$ and $k \leq 0$, then

$$
\begin{aligned}
(\beta- & \left.\|b\|_{\infty}-\left(\int_{0}^{1}|a(t)|^{\frac{\theta+1}{\theta}} d t\right)^{\frac{\theta}{\theta+1}}\right) \int_{0}^{1}\left|x^{\prime}(t)\right|^{\theta+1} d t \\
& \leq\left(\int_{0}^{1}(|p(t)|+|r(t)|)^{\frac{\theta+1}{\theta}} d t\right)^{\frac{\theta}{\theta+1}}\left(\int_{0}^{1}\left|x^{\prime}(t)\right|^{\theta+1} d t\right)^{\frac{1}{\theta+1}} .
\end{aligned}
$$

If $\theta>1$ and $k>0$, then

$$
\begin{aligned}
& \left(\beta-\|b\|_{\infty}-\left(\int_{0}^{1}|a(t)|^{\frac{\theta+1}{\theta}} d t\right)^{\frac{\theta}{\theta+1}}\right) \int_{0}^{1}\left|x^{\prime}(t)\right|^{\theta+1} d t \\
& \quad \leq\left(\int_{0}^{1}(|p(t)|+|r(t)|)^{\frac{\theta+1}{\theta}} d t\right)^{\frac{\theta}{\theta+1}}\left(\int_{0}^{1}\left|x^{\prime}(t)\right|^{\theta+1} d t\right)^{\frac{1}{\theta+1}}+k\left(\int_{0}^{1}\left|x^{\prime}(t)\right|^{\theta+1} d t\right)^{\frac{2}{\theta+1}} .
\end{aligned}
$$


It follows from $\left(A_{2}\right)$ that there is a constant $M>0$ such that $\int_{0}^{1}\left|x^{\prime}(t)\right|^{\theta+1} d t \leq M$.

It is easy from $\left(A_{3}\right)$ to get

$$
\begin{aligned}
& \delta \int_{0}^{1}\left|x^{\prime \prime}(t)\right|^{p} d t \leq \int_{0}^{1} q(t) \phi\left(x^{\prime \prime}(t)\right) x^{\prime \prime}(t) d t \\
& =\phi(\lambda) \lambda\left(k \int_{0}^{1}\left[x^{\prime}(t)\right]^{2} d t+\int_{0}^{1} g\left(t, x(t), x^{\prime}(t)\right) x^{\prime}(t) d t-\int_{0}^{1} p(t) x^{\prime}(t) d t\right) \\
& \leq|k| \int_{0}^{1}\left[x^{\prime}(t)\right]^{2} d t+\int_{0}^{1} h\left(t, x(t), x^{\prime}(t)\right) x^{\prime}(t) d t+\int_{0}^{1}\left|f\left(t, x(t), x^{\prime}(t)\right)\right|\left|x^{\prime}(t)\right| d t \\
& +\int_{0}^{1}\left|p(t) \| x^{\prime}(t)\right| d t \\
& \leq|k| \int_{0}^{1}\left[x^{\prime}(t)\right]^{2} d t+\int_{0}^{1}\left|f\left(t, x(t), x^{\prime}(t)\right)\left\|x^{\prime}(t)\left|d t+\int_{0}^{1}\right| p(t)\right\| x^{\prime}(t)\right| d t \\
& \leq|k| \int_{0}^{1}\left[x^{\prime}(t)\right]^{2} d t+\int_{0}^{1}\left|p(t) \| x^{\prime}(t)\right| d t \\
& +\int_{0}^{1}|a(t)||x(t)|^{\theta}\left|x^{\prime}(t)\right| d t+\left.\int_{0}^{1}|b(t)| x^{\prime}(t)\right|^{\theta+1} d t+\int_{0}^{1}|r(t)|\left|x^{\prime}(t)\right| d t \\
& \int\left[|k|+\left(\int_{0}^{1}|a(t)|^{2} d t\right)^{\frac{1}{2}}+\|b\|_{\infty}\right] \int_{0}^{1}\left|x^{\prime}(t)\right|^{2} d t \\
& \leq\left\{\begin{array}{c}
+\left(\int_{0}^{1}(|p(t)|+|r(t)|)^{2} d t\right)^{\frac{1}{2}}\left(\int_{0}^{1}\left|x^{\prime}(t)\right|^{2} d t\right)^{\frac{1}{2}}, \\
|k|\left(\int_{0}^{1}\left|x^{\prime}(t)\right|^{\theta+1} d t\right)^{\frac{2}{\theta+1}}+\left(\int_{0}^{1}(|p(t)|+|r(t)|)^{\frac{\theta+1}{\theta}} d t\right)^{\frac{\theta}{\theta+1}} \int_{0}^{1}\left|x^{\prime}(t)\right|^{\theta+1} d t
\end{array}\right. \\
& +\left(\int_{0}^{1}|a(t)|^{\frac{\theta+1}{\theta}} d t\right)^{\frac{\theta}{\theta+1}}\left(\int_{0}^{1}\left|x^{\prime}(t)\right|^{\theta+1} d t\right)^{\frac{1}{\theta+1}} \\
& +\|b\|_{\infty} \int_{0}^{1}\left|x^{\prime}(t)\right|^{\theta+1} d t . \\
& \leq\left\{\begin{array}{c}
{\left[|k|+\left(\int_{0}^{1}|a(t)|^{2} d t\right)^{\frac{1}{2}}+|| b \|_{\infty}\right] M+\left(\int_{0}^{1}(|p(t)|+|r(t)|)^{2} d t\right)^{\frac{1}{2}} M^{\frac{1}{2}},} \\
|k| M^{\frac{2}{\theta+1}}+\left(\int_{0}^{1}(|p(t)|+|r(t)|)^{\frac{\theta+1}{\theta}} d t\right)^{\frac{\theta}{\theta+1}} M^{\frac{1}{\theta+1}} \\
+\left(\int_{0}^{1}|a(t)|^{\frac{\theta+1}{\theta}} d t\right)^{\frac{\theta}{\theta+1}} M+\|b\|_{\infty} M .
\end{array}\right.
\end{aligned}
$$

Hence there is a constant $M_{1}>0$ such that $\int_{0}^{1}\left|x^{\prime \prime}(t)\right|^{p} d t \leq M_{1}$. It follows that $\left|x^{\prime}(t)\right| \leq \int_{0}^{1}\left|x^{\prime \prime}(t)\right| d t \leq$ $\left(\int_{0}^{1}\left|x^{\prime \prime}(t)\right|^{p} d t\right)^{\frac{1}{p}} \leq M_{1}^{\frac{1}{p}}$ for all $t \in[0,1]$. Thus $|x(t)| \leq \int_{0}^{1}\left|x^{\prime}(t)\right| d t \leq M_{1}^{\frac{1}{p}}$ for all $t \in[0,1]$. and $\left|x^{\prime}(t)\right| \leq M_{1}^{\frac{1}{p}}$ for all $t \in[0,1]$.

Since $x^{\prime}(0)=x^{\prime}(1)=0$, there is $\xi \in[0,1]$ such that $y(\xi)=0$. Then

$$
\begin{aligned}
|q(t) y(t)| & =\left|q(\xi) y(\xi)+\int_{\xi}^{t}(q(s) y(s))^{\prime} d s\right| \\
& \leq|k| \int_{0}^{1}\left|x^{\prime}(t)\right| d t+\int_{0}^{1}\left|g\left(t, x(t), x^{\prime}(t)\right)\right| d t+\int_{0}^{1}|p(t)| d t
\end{aligned}
$$




$$
\leq|k| M_{1}^{\frac{1}{p}}+\max _{t \in[0,1],|x| \leq M_{1}^{\frac{1}{p}},|y| \leq M_{1}^{\frac{1}{p}}}|g(t, x, y)|+\int_{0}^{1}|p(t)| d t .
$$

Hence

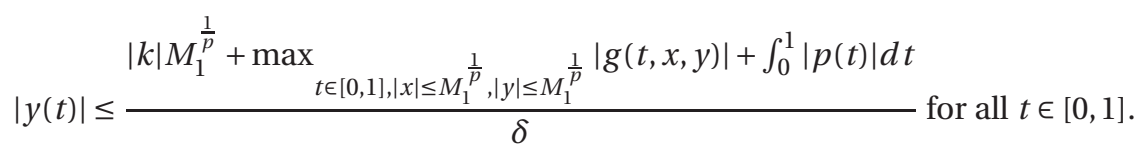

Let

$$
\Omega_{1}=\{(x, y) \in D(L) \bigcap X: L(x, y)=\lambda N(x, y), \lambda \in(0,1)\} .
$$

It follows from above discussion that $\Omega_{1}$ is bounded.

Now, for $(x, y)=(0, c / q(t)) \in \operatorname{Ker} L$, if $N(0, c / q(t)) \in \operatorname{Im} L$, then $\int_{0}^{1} \phi^{-1}(c / q(t)) d t=0$, it follows that $c=0$. Let

$$
\Omega_{2}=\{(x, y) \in \operatorname{Ker} L: N(x, y) \in \operatorname{Im} L\} .
$$

Then $\Omega_{2}=\{0\}$.

Let

$$
\Omega_{3}=\{x \in \operatorname{Ker} L: \lambda \wedge x+(1-\lambda) Q N x=0, \lambda \in[0,1]\},
$$

where $\wedge: Y / \operatorname{Im} L \rightarrow \operatorname{Ker} L$ is given by $\wedge^{-1}(c, 0)=(0, c / q(t))$. For $(0, c / q(t)) \in \Omega_{3}$, and $\lambda \in[0,1]$, we have

$$
-(1-\lambda) Q(\phi(c / q(t)), f(t, 0,0))=\lambda \wedge(0, c / q(t)) .
$$

It follows that

$$
-(1-\lambda) \int_{0}^{1} \phi(c / q(t)) d t=\lambda c .
$$

If $\lambda=1$, then $c=0$. If $\lambda \in[0,1)$, then

$$
0 \leq \lambda c^{2}=-(1-\lambda) c \phi(c) \int_{0}^{1} \phi(1 / q(t)) d t<0,
$$

a contradiction. So $\Omega_{3}=\{0\}$.

Let $X$ and $Y$ be Banach spaces, $L: D(L) \subset X \rightarrow Y$ be a Fredholm operator of index zero, $P: X \rightarrow X, Q: Y \rightarrow Y$ be projectors such that

$$
\operatorname{Im} P=\operatorname{Ker} L, \operatorname{Ker} Q=\operatorname{Im} L, X=\operatorname{Ker} L \oplus \operatorname{Ker} P, Y=\operatorname{Im} L \oplus \operatorname{Im} Q .
$$

It follows that

$$
\left.L\right|_{D(L) \cap \operatorname{Ker} P}: D(L) \cap \operatorname{Ker} P \rightarrow \operatorname{Im} L
$$

is invertible, we denote the inverse of that map by $K_{p}$.

If $\Omega$ is an open bounded subset of $X, D(L) \cap \bar{\Omega} \neq \varnothing$, the map $N: X \rightarrow Y$ will be called $L$-compact on $\bar{\Omega}$ if $Q N(\bar{\Omega})$ is bounded and $K_{p}(I-Q) N: \bar{\Omega} \rightarrow X$ is compact.

Theorem 2.1.([5]) Let L be a Fredholm operator of index zero and let $N$ be L-compact on $\Omega$. Assume that the following conditions are satisfied: 
(i) $L x \neq \lambda N x$ for every $(x, \lambda) \in[(\operatorname{dom} L \backslash \operatorname{Ker} L) \cap \partial \Omega] \times(0,1)$;

(ii) $N x \notin \operatorname{ImL}$ for every $x \in \operatorname{Ker} L \cap \partial \Omega$;

(iii) $\operatorname{deg}\left(\left.\wedge Q N\right|_{\text {KerL }}, \Omega \cap \operatorname{KerL}, 0\right) \neq 0$, where $\wedge: Y / \operatorname{ImL} \rightarrow$ KerL is the isomorphism.

Then the equation $L x=N x$ has at least one solution in $D(L) \cap \bar{\Omega}$.

Theorem L. Suppose that $\left(A_{1}\right)-\left(A_{3}\right)$ hold. Then problem (3) has at least one solution.

Proof. Set $\Omega$ be a open bounded subset of $X$ such that $(0,0) \in \Omega \supseteq \bar{\Omega}_{1}$. We know that $L$ is a Fredholm operator of index zero and $N$ is $L$-compact on $\bar{\Omega}$. By the definition of $\Omega$, we have $L x \neq \lambda N x$ for $x \in(D(L) / \operatorname{Ker} L) \cap \partial \Omega$ and $\lambda \in(0,1) ; N x \notin \operatorname{Im} L$ for $x \in \operatorname{Ker} L \cap \partial \Omega$.

In fact, let $H(x, \lambda)=\lambda \wedge x+(1-\lambda) Q N x$. According the definition of $\Omega$, we know $\Omega \supset \bar{\Omega}_{3}$, thus $H(x, \lambda) \neq 0$ for $x \in \partial \Omega \cap \operatorname{Ker} L$, thus by homotopy property of degree,

$$
\begin{aligned}
& \operatorname{deg}(Q N \mid \operatorname{Ker} L, \Omega \cap \operatorname{Ker} L, 0)=\operatorname{deg}(H(\cdot, 0), \Omega \cap \operatorname{Ker} L, 0) \\
& \quad=\operatorname{deg}(H(\cdot, 1), \Omega \cap \operatorname{Ker} L, 0)=\operatorname{deg}(\wedge, \Omega \cap \operatorname{Ker} L, 0) \neq 0 .
\end{aligned}
$$

Thus by Theorem 2.1, $L(x, y)=N(x, y)$ has at least one solution in $D(L) \cap \bar{\Omega}$, then $x$ is a solution of problem (3). The proof is completed.

\section{Examples}

In this section, we present an example to illustrate the main result.

Example 3.1. Consider the problem

$$
\left\{\begin{array}{l}
{\left[\left(t^{2}+2\right) x^{\prime \prime}(t)\right]^{\prime}+k x^{\prime}(t)=\beta \frac{3\left[x^{\prime}(t)\right]^{2 l+1}}{2+\sin [x(t)]^{2}}+a(t)[x(t)]^{2 l+1}+b(t)\left[x^{\prime}(t)\right]^{2 l+1}+r(t), t \in(0,1),} \\
x^{(i)}(0)=x^{(i)}(1), \quad i=0,1,2,
\end{array}\right.
$$

where $\beta>0, k \in R, l$ is a positive integer, $a, b, r \in L^{1}[0,1]$. Corresponding to problem (3), let $\phi(x)=x, g(t, x, y)=-\beta \frac{3 y^{2 l+1}}{2+\sin x^{2}}-a(t) x^{2 l+1}-b(t) y^{2 l+1}-r(t), p(t)=0$ and set

$$
h(t, x, y)=-\beta \frac{3 y^{2 l+1}}{2+\sin x^{2}}, f(t, x, y)=-a(t) x^{2 l+1}-b(t) y^{2 l+1}-r(t) .
$$

It is easy to show from Theorem $\mathrm{L}$ that problem (5) has at least one solution if $l>0$, and

$$
\|b\|_{\infty}+\left(\int_{0}^{1}|a(t)|^{\frac{\theta+1}{\theta}} d t\right)^{\frac{\theta}{\theta+1}}<\beta
$$

or if $l=0$ and

$$
k+\|b\|_{\infty}+\left(\int_{0}^{1}|a(t)|^{2} d t\right)^{\frac{1}{2}}<\beta .
$$

The equation in problem (5) can be transformed into

$$
x^{\prime \prime \prime}(t)+\frac{2 t}{t^{2}+2} x^{\prime \prime}(t)+\frac{k}{t^{2}+2} x^{\prime}(t)=\frac{\beta\left[x^{\prime}(t)\right]^{2 l+1}+a(t)[x(t)]^{2 l+1}+b(t)\left[x^{\prime}(t)\right]^{2 l+1}+r(t)}{t^{2}+2} .
$$


It is easy to find that $\left(B_{1}\right),\left(B_{2}\right),\left(B_{3}\right),(C)$ are not satisfied. So Example 3.1 can not be solved by theorems in papers [1-4].

Example 3.2. Consider the problem

$$
\left\{\begin{array}{l}
{\left[\left(x^{\prime \prime}(t)\right)^{3}\right]^{\prime}=\beta\left[x^{\prime}(t)\right]^{3}+a(t)[x(t)]^{3}+b(t)\left[x^{\prime}(t)\right]^{3}+r(t), t \in(0,1),} \\
x^{(i)}(0)=x^{(i)}(1), \quad i=0,1,2
\end{array}\right.
$$

where $\beta>0, l$ is a positive integer, $a, b, r \in L^{1}[0,1], \phi(x)=|x|^{2} x$. It is easy to find that problem (6) has at least one solution if

$$
\|b\|_{\infty}+\left(\int_{0}^{1}|a(t)|^{\frac{4}{3}} d t\right)^{\frac{3}{4}}<\beta
$$

\section{References}

[1] R. Ma, Multiplicity results for a third order boundary value problem at resonance, Nonl. Anal. TMA 32(1998), 493-499.

[2] P. K. Nagle and K. L. Pothoven, On a third order nonlineqar boundary value problem at resonance, J. Math. Anal. Appli., 195(1995), 149-159.

[3] C. P. Gupta, On a third order boundary value problem at resonance, Differential Integral Equations 2(1989), 1-12.

[4] E. Rovderova, Third-order boundary value problem with nonlinear boundary conditions, Nonl. Anl. 25(1995), 473-485.

[5] J. Mawhin, Topological degree and boundary value problems for nonlinear differential equations, in: P. M. Fitzpertrick, M. Martelli, J. Mawhin, R. Nussbanm(Eds.), Topological Methods for Ordinary Differential Equations, Lecture Notes in Math. Vol.1537, Springer-Verlag, New York/Berlin, 1991.

Department of Mathematics, Shaoyang University, Hunan 422600, P.R. China.

E-mail: liuyuji888@sohu.com

Department of Mathematics, Guangdong University of Business Studies, Guangzhou 510320, P.R. China. 